\title{
Magnetic Properties of Polymer Containing Verdazyl in the Side Chain
}

\author{
Mikiharu KaMACHI, Hiroshi ENOMOTO, Mahito SHIBASAKA, \\ Wasuke MORI, ${ }^{*}$ and Michihiko KISHITA* \\ Department of Macromolecular Science, Faculty of Science, \\ Osaka University, Toyonaka, Osaka 560, Japan \\ * Department of Chemistry, College of General Education, \\ Osaka University, Toyonaka, Osaka 560, Japan
}

(Received December 9, 1985)

\section{KEY WORDS Magnetic Property / Verdazyl / ESR / Magnetic Susceptibility / Antiferromagnetic Interaction / Electron Exchange /}

ESR spectra of polymers containing paramagnetic species in their side chains show that electron exchange interactions take place between paramagnetic side chains. ${ }^{1-3}$ These phenomena suggest the possibility that the organic polymers containing paramagnetic species might be a new type of magneitc material owing to the magnetically long-range ordering of unpaired electron through spinspin interactions. Based on this idea, we compared the temperature dependence of the magnetic susceptibility of a polymer containing the 2,2,6,6-tetramethyl pyperidin-1-oxyl group (poly(MOTMP)) with that of the corresponding monomeric compound. ${ }^{1} \mathrm{~A}$ weak antiferromagnetic interaction was observed in the polymer, while there was no recognizable magnetic interaction between the neighboring paramagnetic species in the monomer. The exchange interaction of electron spins between nitroxyls was not so strong in the polymer as expected. This is possibly due to the fact that the distance between the neighboring nitroxyl groups is too far for the unpaired electrons to interact with each other through the side chains, because an unpaired electron of the nitroxyl is localized on the $\mathrm{N}-\mathrm{O}$ bond. A paramagnetic side group whose electron is more delocalized is preferable to obtain a magnetically interacting polymer. Since an unpaired electron of the verdazyl is known to be delocalized on the

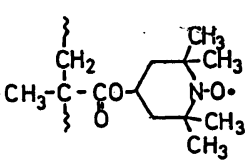

Poly(MOTMP)

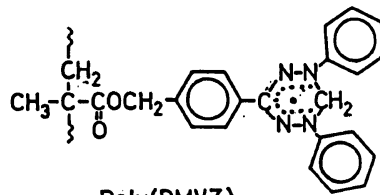

Poly(DMVZ) verdazyl ring, ${ }^{4}$ we prepared a polymer containing verdazyl in the side chain.

In this paper, we compare the magnetic susceptibility of the polymer containing the verdazyl radical in the side chain with that of the corresponding monomeric one.

\section{EXPERIMENTAL}

\section{Materials}

1,5-Diphenyl-3-( $p$-methacryloyloxymethylphenyl)formazane was synthesized according to the literature ${ }^{5}$ starting from methacryl chloride and 1,5-diphenyl-3-( $p$-hydroxymeth- 
ylphenyl)formazane which was prepared by terephthalaldehyde and phenylhydrazine. ${ }^{5}$ Reddish brown needles of $\mathrm{mp} 103-105^{\circ} \mathrm{C}$ were obtained in $81 \%$ yield.

1,5-Diphenyl-3-( $p$-methacryloyloxymethylphenyl)verdazyl (DMVZ) was prepared according to the literature, ${ }^{5}$ starting from 1,5 diphenyl-3-( $p$-hydroxymethylphenyl)formazane, $37 \%$ formaldehyde, and phenylhydrazine. Dark green needles of $\mathrm{mp} 124-126^{\circ} \mathrm{C}$ were obtained in $84 \%$ yield, $\lambda_{\text {max }} ; 730 \mathrm{~nm}^{5}$

Poly(DMVZ) was prepared by the polymerization of DMVZ with butyllithium in THF at $0^{\circ} \mathrm{C}$. The polymer was precipitated with diethyl ether, and reprecipitated twice with benzene-diethyl ether. The molecular weight was determined to be 4500 by vapor pressure osmometry.

\section{Measurement}

ESR spectra were measured with a Japan Electrom Optics Laboratory Model JEOL FE-1X ESR spectrometer with a $100 \mathrm{KHz}$ modulator.

Gram magnetic susceptibility $\left(\chi_{\mathrm{g}}\right)$ was determined by the Faraday method using a Cahn balance in a temperature range from 4 to $273 \mathrm{~K}$. The molar magnetic susceptibility $\left(\chi_{\mathrm{M}}=\chi_{\mathrm{g}} \times M\right.$, where $M$ is the molecular weight of DMVZ or monomer unit of the polymer) was corrected for diamagnetic contributions of atoms with their Pascal constants ${ }^{6}$; the $\chi_{\text {dia }}$ was $-350 \times 10^{-6}$ cgsemu.

\section{RESULTS AND DISCUSSION}

The ESR spectra of DMVZ and poly(DMVZ) are shown in Figures 1 and 2. In the case of DMVZ the line shape depends on the concentration of the radical. A nine line spectrum due to four nitrogen nuclei, which was observed at $10^{-4} \mathrm{M}$, became broader on increasing the concentration of the radical, and then coalesced to a single line at $1.0 \mathrm{M}$. The ESR spectra of poly(DMVZ) showed a broad line with nine shoulders even at $10^{-4} \mathrm{M}$ of the
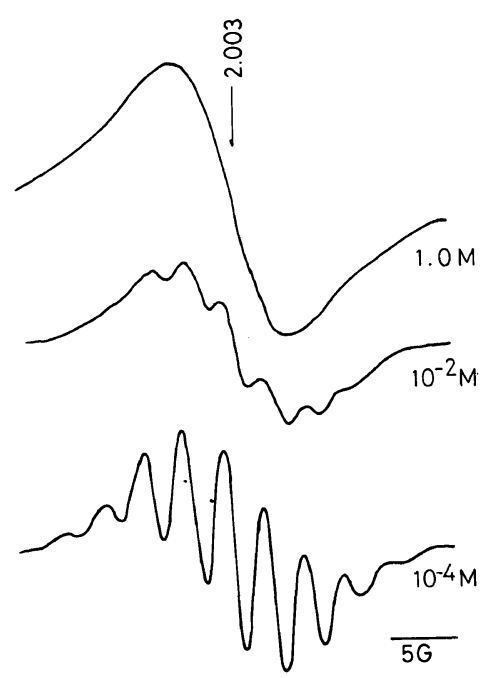

Figure 1. ESR spectra of DMVZ at various concentrations.
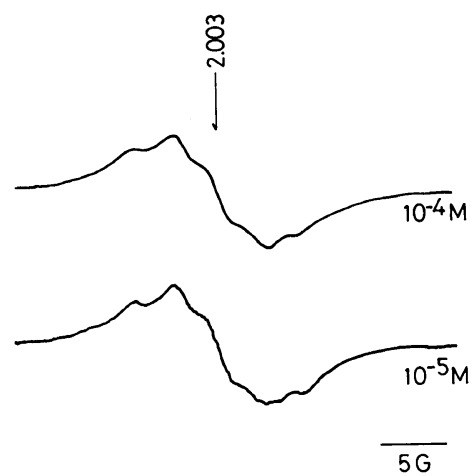

Figure 2. ESR spectra of poly(DMVZ) monomer unit concentrationat $10^{-4} \mathrm{M}$ and $10^{-5} \mathrm{M}$.

radical unit, and the line was independent of concentration. Thus, electron exchange between the paramagnetic side chains of poly(DMVZ) was found to be much easier than the intermolecular exchange of DMVZ.

The temperature dependences of the reciprocal of magnetic susceptibility of poly(DMVZ) and DMVZ are shown in Figure 3. An extraporation from the high temperature region gave a positive intercept on the ordinate for $\mathrm{DMV}$, indicating that the temperature dependence of $\chi_{M}$ follows the Curie-Weiss law with $\theta=-21.9 \mathrm{~K}$. 


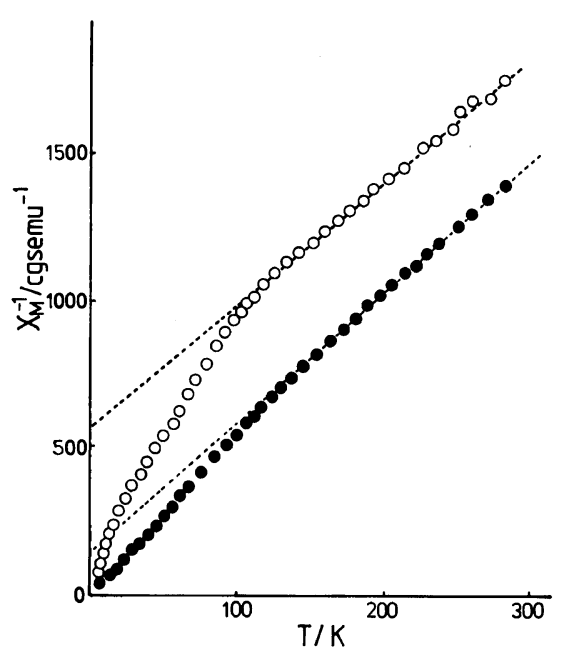

Figure 3. Relation between the reciprocal of magnetic susceptibility and temperature: $(\bigcirc)$ poly(DMVZ); DMV.Z.

For poly(DMVZ), a straight line across a higher value of the positive ordinate was observed when extraporated, indicating that $\chi_{M}$ obey the Curie-Weiss law with $\theta=-150 \mathrm{~K}$. The results show that a stronger antiferromagnetic interaction takes place between verdazyl groups in a polymer chain as compared to the corresponding monomeric compound. The lower values of $\chi_{M}$ in the polymer as compared to the monomer support the occurrence of antiferromagnetic interactions. The degree of antiferromagnetic interaction of the verdazyl group in the polymer is larger than that of in the nitroxyl group $(\theta=-6.6 \mathrm{~K})$. This difference may be due to differences in the delocalizability of the unpaired electron of verdazyl and nitroxyl.

The antiferromagnetic interaction of poly(DMVZ). is much weaker than that of the polymer containing porphyrin-Ag(II), ${ }^{2,3}$ whose Neel point is about $0^{\circ} \mathrm{C}$, but, to our knowledge, it is the strongest in the organic polymers. ${ }^{1-3}$ These results may possibly serve in the molecular design of a polymer magnet.

Acknowledgments. The authors thank Professor S. Nozakura for his helpful and numerous comments for the preparation of this manuscript.

\section{REFERENCES}

1. M. Kamachi, M. Tamaki, Y. Morishima, S. Nozakura, W. Mori, and M. Kishita, Polym. J., 14, 363 (1982).

2. M. Kamachi, H. Akimoto, W. Mori, and M. Kishita, Polym. J., 16, 23 (1984).

3. S. Nozakura and M. Kamachi, Makromol. Chem., Suppl., 12; 255 (1985).

4. O. M. Polumbrik, Russian Chem. Rev., 47, 767 (1978).

5. Y. Miura, M. Kinoshita, and M. Imoto, Makromol. Chem., 157, 51 (1972).

6. F. E. Mabbs and D. J. Machin, "Magnetism and Transition Metal Complexes," Chapman and Hall, London, 1973, p 5. 